\title{
Hepatopatia crônica associada a tratamento prolongado com fenobarbital em caninos
}

\section{Chronic hepatopathy in dogs due to long-term treatment with Phenobarbital}

\author{
Mariana Boscato Menegat ${ }^{1}$; Angelica Terezinha Barth Wouters ${ }^{1 *}$; \\ Flademir Wouters ${ }^{1}$; Tatiane Terumi Negrão Watanabe ${ }^{1}$; \\ Suyene Oltramari Souza ${ }^{1}$; David Driemeier ${ }^{2}$
}

\begin{abstract}
Resumo
Doenças hepáticas crônicas são frequentes em caninos e, muitas vezes, não tem causa determinada. Elas têm sido relacionadas com terapia prolongada com fenobarbital, fármaco usado com frequência na rotina da clínica de pequenos animais, sendo importante a verificação do uso prolongado de fenobarbital na investigação da causa da hepatopatia. Foi realizado um estudo retrospectivo do arquivo de necropsias do Setor de Patologia Veterinária da Universidade Federal do Rio Grande do Sul no período de 2000 a 2010 e identificados dois casos de hepatopatia crônica em caninos com histórico de uso de fenobarbital por período superior a seis meses. Os achados mais relevantes nos exames de necropsia foram fígado reduzido de volume, firme e com superfície irregular; ascite e edema em outras localizações; além de desvios vasculares portossistêmicos. No exame histológico do fígado havia fibrose difusa, proliferação de ductos biliares, infiltrado inflamatório mononuclear, colestase e, em um dos casos, nódulos de regeneração com vacuolização hepatocelular. Os achados macro e microscópicos determinaram o diagnóstico de hepatopatia crônica e a intensidade das lesões foi condizente com o tempo de uso do fenobarbital.
\end{abstract}

Palavras-chave: Doenças do fígado, patologia, pequenos animais, anticonvulsivantes

\begin{abstract}
Chronic liver diseases are common in dogs, however, their causes are often undetermined. They have been associated with long-term phenobarbital therapy, used in large-scale as anticonvulsivant drug in small animal clinics. In the investigation of etiology for liver disease in dogs it's important to check the prolonged use of phenobarbital. A retrospective study of canine necropsies performed at Laboratory of Veterinary Pathology of Universidade Federal do Rio Grande do Sul, was made from 2000 to 2010, and two cases of chronic diffuse hepatopathy in dogs under phenobarbital therapy (more than six months of treatment) were diagnosed. The main macroscopic findings, such as smaller, firmer and irregular surface of liver, ascites and other sites edema, and portosystemic shunts were observed. Histologically, the most important lesions were observed in the liver. There were diffuse fibrosis, bile ducts proliferation, mononuclear inflammatory infiltrate, cholestasis, and, in one case also occurred regenerating nodules of hepatocytes with vacuolization of hepatocytes cytoplasm. The diagnosis of chronic hepatopathy was based on these findings, and the intensity of the injuries was consistent with the time of treatment using phenobarbital.
\end{abstract}

Key words: Liver diseases, pathology, small animals, anticonvulsants

\footnotetext{
${ }^{1}$ Discentes, Universidade Federal do Rio Grande do Sul, UFRGS, Porto Alegre, RS. E-mail: mariana_menegat@hotmail.com; angelicawouters@yahoo.com.br; flademir@dmv.ufla.br; beijos.tati@hotmail.com; suy.souza@hotmail.com

${ }^{2}$ Prof. e Pesquisador, UFRGS, Porto Alegre, RS. E-mail: davetpat@ufrgs.br

* Autor para correspondência
} 


\section{Introdução}

Fenobarbital é considerado o medicamento de escolha para o tratamento inicial e continuado de convulsões (TAYLOR, 2010; JOHNSON; SHERDING, 2008) por ser um anticonvulsivante relativamente seguro, eficaz e barato (TAYLOR, 2010). No entanto, os fármacos anticonvulsivantes têm sido associados ao desenvolvimento de doença hepática crônica e cirrose em caninos (BUNCH et al., 1982, 1984; DAYRELL-HART et al., 1991; STALKER; HAYES, 2007; CULLEN; BROWN, 2012). Cirrose é a denominação usada para o estágio terminal da hepatopatia crônica, caracterizada por fibrose difusa, nódulos de regeneração e proliferação de ductos biliares (STALKER; HAYES, 2007; CRAWFORD, 2010; BARROS, 2011; CULLEN; BROWN, 2012). No entanto, muitos patologistas consideram o termo cirrose demasiadamente impreciso para ser utilizado como um diagnóstico morfológico e tendem a evitar seu uso (STALKER; HAYES, 2007; CULLEN; BROWN, 2012).

O objetivo deste trabalho é descrever dois casos de hepatopatia crônica em caninos com histórico de uso prolongado de fenobarbital.

\section{Material e Métodos}

Foi realizado levantamento de dados do arquivo de necropsias do Setor de Patologia Veterinária da Universidade Federal do Rio Grande do Sul (SPVUFRGS), dos casos de hepatopatia crônica em caninos e dos casos com histórico de medicação prolongada com fenobarbital, no período de janeiro de 2000 a dezembro de 2010. Foram avaliados os históricos e os achados de necropsia e efetuada a avaliação histológica dos casos em Hematoxilina e Eosina (H.E.), bem como foram realizadas e avaliadas as colorações de Tricrômico de Masson para evidenciação de tecido conjuntivo fibroso; Ácido Rubeânico para cobre; e Perls para ferro (PROPHET et al., 1996).

\section{Resultados, Discussão e Conclusões}

De janeiro de 2000 a dezembro de 2010 foram diagnosticados no SPV-UFRGS 62 cães com hepatopatia difusa crônica. Desses casos, dois caninos, fêmeas, tinham histórico de tratamento com fenobarbital por, no mínimo, sete meses. As características dos animais, os achados clínicos e de patologia clínica são apresentados na Tabela 1 .

Tabela 1. Dados gerais, tempo de medicação com fenobarbital, achados clínicos e de patologia clínica dos caninos com lesões de hepatopatia crônica.

\begin{tabular}{cccccc}
\hline Canino & Raça & $\begin{array}{c}\text { Idade } \\
(\mathrm{anos})\end{array}$ & $\begin{array}{c}\text { Tratamento fenobarbital } \\
\text { (meses) }\end{array}$ & $\begin{array}{c}\text { Achados } \\
\text { clínicos }\end{array}$ & $\begin{array}{c}\text { Achados patologia } \\
\text { clínica }\end{array}$ \\
\hline 1 & Dachshund & 4 & 7 & $\begin{array}{c}\text { Anasarca, } \\
\text { icterícia, } \\
\text { decúbito } \\
\text { Anasarca, } \\
\text { decúbito }\end{array}$ & $\uparrow \mathrm{FA}, \uparrow \mathrm{Bb}$ total \\
2 & Poodle & 11 & 96 & $\begin{array}{c}\downarrow \text { albumina, } \downarrow \text { glicose, } \\
\uparrow \text { ALT }\end{array}$ \\
\hline
\end{tabular}

$\mathrm{FA}=$ fosfatase alcalina; $\mathrm{Bb}=$ bilirrubina $\mathrm{ALT}=$ alanina transaminase.

Fonte: Elaboração dos autores.

Ao exame macroscópico, o fígado dos dois animais estava diminuído de tamanho, firme, com superfície irregular (Canino 1) ou nodular (Canino 2; Figura 1; A). As lesões extra-hepáticas encontradas foram icterícia (Canino 1), ascite (Canino 2), desvios vasculares portossistêmicos (Caninos 1 e 2), esplenomegalia discreta (Canino
2) a moderada (Canino 1), edema subcutâneo moderado (Canino 1) a acentuado (Canino 2), hidrotórax (Caninos 1 e 2), hidropericárdio (Canino 1), edema pulmonar (Caninos 1 e 2), úlceras gástricas (Canino 2) e conteúdo sanguinolento no intestino grosso (Canino 1). 
Figura 1. Hepatopatia crônica associada a tratamento prolongado com fenobarbital em caninos.

A. Necropsia do Canino 2 - fígado com acentuada redução de volume e superfície nodular. B. Fígado do Canino $2-$ fibrose (cabeça de seta), proliferação de ductos biliares (setas), nódulo de regeneração com vacuolização hepatocelular (asterisco) e infiltrado inflamatório predominantemente mononuclear (estrela). H.E. Obj.10x. C. Fígado do Canino 1- fibrose com proliferação de ductos biliares (setas), desorganização de cordões de hepatócitos (asterisco) e pigmento amarelo-acastanhado. H.E. Obj. 20x. D. Fígado do Canino 2- fibrose (em azul) entremeada por proliferação de ductos biliares. Tricrômico de Masson. Obj. 20x.
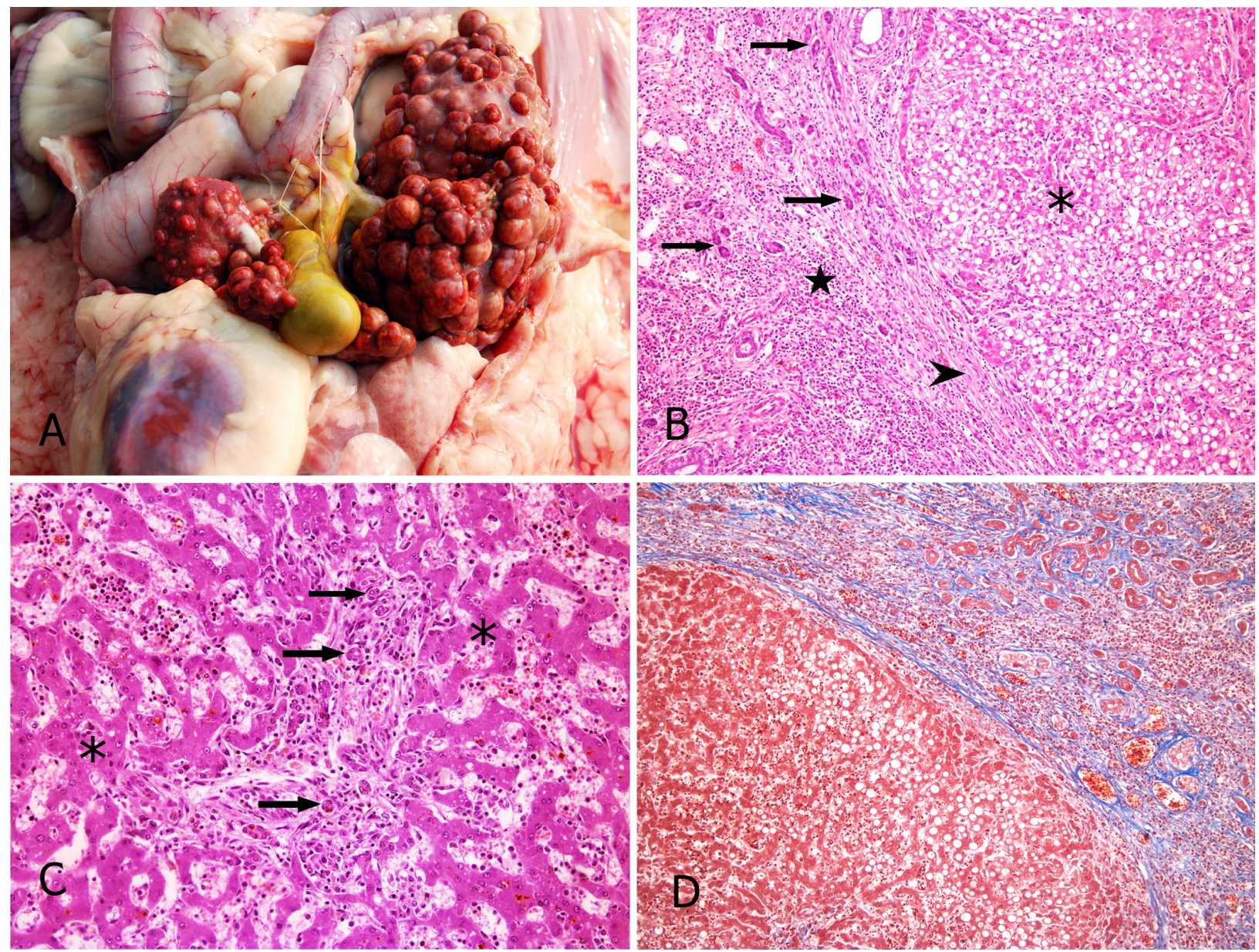

Fonte: Elaboração dos autores.

$\mathrm{Na}$ avaliação histológica do fígado, em ambos os casos havia fibrose difusa, moderada (Canino 1) ou acentuada (Canino 2) (Figura 1; B e D); proliferação de ductos biliares (Figura 1; B e C) discreta (Canino 1) ou acentuada (Canino 2); colestase intra-hepática discreta (Canino 2) ou moderada (Canino 1); nódulos de regeneração (Canino 2, Figura 1; B); distorção dos cordões de hepatócitos (Canino 1, Figura 1C), associada à dilatação de sinusoides hepáticos; e infiltrado inflamatório, predominantemente mononuclear
(Figura 1; B) discreto (Canino 1) ou moderado (Canino 2), com distribuição principalmente portal. Pigmento granular amarelo-acastanhado em hepatócitos e células de Kupffer foi observado em um dos casos (Canino 2) e vacuolização bem definida no citoplasma dos hepatócitos nos nódulos de regeneração era evidente no Canino 2 (Figura 1; B). A coloração de Tricrômico de Masson evidenciou o tecido conjuntivo fibroso proliferado no fígado de ambos os casos (Figura 1D). O método histoquímico de Perls, corou o citoplasma de hepatócitos e 
células de Kupffer (Canino 2). Nenhum dos fígados dos caninos em estudo teve marcação positiva na coloração por Ácido Rubeânico.

Os achados de necropsia e histológicos evidenciaram insuficiência hepática crônica como causa da morte dos animais. Icterícia, ascite, hidrotórax, edema subcutâneo e desvios portossistêmicos, com variação em frequência e intensidade, são as alterações macroscópicas associadas à insuficiência hepática crônica (STALKER; HAYES, 2007; CULLEN; BROWN, 2012). A vulnerabilidade do fígado a injúrias se deve principalmente à frequente exposição a drogas, tanto por fármacos como por metabólitos de biotransformação hepática (BARROS, 2011) e a toxicidade crônica por agentes terapêuticos está entre as causas potenciais de hepatopatia crônica (CULLEN; BROWN, 2012), no entanto, apesar de relatada em cães, não tem prevalência determinada (BARROS, 2011). Como os animais estavam sob tratamento com fenobarbital por período superior a seis meses e o fenobarbital é uma droga reconhecidamente hepatotóxica (BUNCH et al., 1982, 1984; DAYRELL-HART et al., 1991), as lesões hepáticas foram atribuídas ao uso do medicamento por tempo prolongado.

As drogas hepatotóxicas são classificadas em duas categorias básicas: a das hepatotoxinas intrínsecas, isto é, que tem efeitos previsíveis e afetam a maioria dos animais expostos; e a das idiossincráticas, com reações inesperadas. Os anticonvulsivantes, usados sozinhos ou em associação com outros medicamentos, podem induzir sinais bioquímicos e clínicos de disfunção hepática em caninos medicados por mais de seis meses. Sua real hepatotoxicidade pode representar uma reação idiossincrática em uma pequena parcela dos cães medicados, mas não pode ser descartada a possibilidade de hepatotoxicidade intrínseca dosedependente nos casos de tratamento prolongado. É também possível que a indução enzimática associada com a terapia anticonvulsivante altere a habilidade hepática de detoxificar outros compostos hepatotóxicos não específicos aos quais os animais estão expostos (STALKER; HAYES, 2007).

As alterações induzidas pelos anticonvulsivantes nem sempre são uniformes, pois seus efeitos podem ser influenciados por uma série de fatores, como idade, sexo, dieta, funções endócrinas e constituição genética (CULLEN; BROWN, 2012). Além disso, deve ser considerada a sensibilidade individual dos animais ao medicamento. É descrito que alguns animais podem desenvolver reações idiossincráticas e sinais de toxicidade acentuada ao fenobarbital (DAYRELL-HART et al., 1991; MÜLLER et al., 2000).

O acúmulo de ferro no fígado, que se caracteriza por coloração acastanhada e aspecto granular na coloração por H.E. (CULLEN; BROWN, 2012) e confirmado em um dos dois caninos desse estudo pela coloração de Perls, é descrito em cães com lesão hepática crônica (FUENTEALBA et al., 1997). Ocorre sob a forma de hemossiderina em hepatócitos e em células de Kupffer e tem relação com excesso sistêmico de ferro ou necrose hepatocelular.

O acúmulo de cobre, uma etiologia conhecida da cirrose hepática em determinadas raças caninas predispostas (BEXFIELD et al., 2012), foi descartado como causa da lesão hepática neste estudo, pois em nenhum fígado foi evidenciado acúmulo de cobre, avaliado pela coloração de Ácido Rubeânico.

Outra causa para hepatopatia crônica a ser considerada em caninos é a ingestão de aflatoxinas. Sua presença na dieta mais provavelmente está relacionada à inclusão de milho no alimento de caninos, um importante substrato para o desenvolvimento de Aspergillus flavus, produtor de micotoxinas (BARROS, 2011). Vários casos de hepatopatia fatal por aflatoxinas em caninos foram registrados em caninos da zona rural do interior do Rio Grande do Sul (WOUTERS et al., 2013) e teve relação com o hábito de proprietários de caninos na zona rural incluírem milho na dieta dos animais (BARROS, 2011). Nos casos de aflatoxicose em 
caninos, um achado consistente é esteatose hepática difusa, tanto em casos agudos, subagudos ou crônicos (BASTIANELLO et al., 1987). Os casos relatados são de caninos mantidos na zona urbana e alimentados com ração comercial e em nenhum deles havia esteatose hepática difusa.

Em caninos, a hepatopatia crônica é considerada doença prevalente em pacientes geriátricos (SILVA et al., 2007; POLDERVAART et al., 2009; BEXFIELD et al., 2012). Neste estudo, um dos animais não era idoso. Essa observação reforça a hipótese da lesão hepática ter sido causada pelo fenobarbital, uma vez que os animais iniciaram o tratamento com anticonvulsivante muito jovens e o mantiveram por período prolongado. Uma forma de hepatopatia diagnosticada em cães jovens é a hepatite lobular dissecante, em que o fígado tem volume diminuído e superfície lisa (BARROS, 2011). Nos casos avaliados, o fígado era multinodular ou tinha superfície irregular.

Dermatite necrolítica superficial, descrita em caninos sob tratamento com fenobarbital (MARCH et al., 2004), não foi evidenciada nos exames macroscópico e histológico dos caninos do estudo. Essa lesão não é especificamente associada à hepatopatia por anticonvulsivantes (STALKER; HAYES, 2007), pois ela pode ocorrer em associação com outras lesões hepáticas. Outros autores afirmam que, raramente, as lesões cutâneas ocorrem simultaneamente às cirróticas (MARCH et al., 2004).

Provavelmente o número de casos de hepatopatia crônica por fenobarbital seja bem maior, no entanto, em muitos dos casos de caninos diagnosticados com hepatopatia crônica não foi possível o acesso ao histórico completo.

Os achados permitem concluir que o fenobarbital é um fármaco com ação hepatotóxica e deve ser considerado na investigação da etiologia da hepatopatia crônica em caninos.

\section{Agradecimentos}

À Coordenação de Aperfeiçoamento de Pessoal de Nível Superior (CAPES) e ao Conselho Nacional de Desenvolvimento Científico e Tecnológico (CNPq).

\section{Referências}

BARROS, C. S. L. Fígado, vias biliares e pâncreas exócrino. In: SANTOS, R. L.; ALESSI, A. C. (Ed.). Patologia veterinária. São Paulo: Roca, 2011. cap. 4, p. 183-290.

BASTIANELLO, S. S.; NESBIT, J. W.; WILLIAMS, M. C.; LANGE, A. L. Pathological findings in a natural outbreak of aflatoxicosis in dogs. Journal of Veterinary Research, Onderstepoort, v. 54, n. 4, p. 635-640, 1987.

BEXFIELD, N. H.; BUXTON, R. J.; VICEK, T. J.; DAY, M. J.; BAILEY, S. M.; HAUGLAND, S. P.; MORRISON, L. R.; ELSE, R. W.; CONSTANTINOCASAS, F.; WATSON, P. J. Breed, age and gender distribution of dogs with chronic hepatitis in the United Kingdom. The Veterinary Journal, London, v. 193, n. 1, p. 124-128, 2012. Disponível em: <http://www.ncbi.nlm. nih.gov/pubmed/22225827>. Acesso em: 06 fev. 2012.

BUNCH, S. E.; BALDWIN, B. H.; HORNBUCKLE, W. E.; TENNANT, B. C. Compromised hepatic function in dogs treated with anticonvulsant drugs. Journal of American Veterinary Medical Association, Illinois, v. 184, n. 4, p. 44-448, 1984.

BUNCH, S. E.; CASTLEMAN, W. L.; HORNBUCKLE, W. E.; TENNANT, B. C. Hepatic cirrhosis associated with long term anticonvulsant drug therapy in dogs. Journal of American Veterinary Medical Association, Illinois, v. 181, n. 4, p. 357-362, 1982.

CRAWFORD, J. M. Liver and biliary tract. In: KUMAR, V.; ABBAS, A. K.; FAUSTO, N.; ASTER, J. (Ed.). Robbins and Cotran pathologic basis of disease. 8. ed. Philadelphia: Elsevier Saunders, 2010. cap. 18, p. 833890.

CULLEN, J. M.; BROWN, D. L. Hepatobiliary system and exocrine pancreas. In: McGAVIN, M. D.; ZACHARY, J. F. (Ed.). Pathologic basis of veterinary disease. 5. ed. St. Louis: Elsevier Mosby, 2012. cap. 8, p. 405-453. 
DAYRELL-HART, B.; STEINBERG, S. A.; VANWINKLE, T. G.; FARNBACH, G. C. Hepatotoxicity of phenobarbital in dogs: 18 cases (1985-1989). Journal of American Veterinary Medical Association, Illinois, v. 199, n. 8, p. 1060-1066, 1991.

FUENTEALBA, C.; GUEST, S.; HAYWOOD, S.; HORNEY, B. Chronic hepatitis: a retrospective study in 34 dogs. Canadian Veterinary Journal, Guelph, v. 38, n. 6, p. 365-373, 1997. Disponível em: <http://www.ncbi. nlm.nih.gov/pmc/articles/PMC1576879>. Acesso em: 06 fev. 2012.

JOHNSON, S. E.; SHERDING, R. G. Doenças do fígado e trato biliar. In: BIRCHARD, S. J.; SHERDING, R. G. (Ed.). Manual Saunders clínica de pequenos animais. 3. ed. São Paulo: Roca, 2008. cap. 71, p. 765-829.

MARCH, P. A.; HILLIER, A.; WEISBRODE, S. E.; MATTOON, J. S.; JOHNSON, S. E.; DIBARTOLA, S. P.; BROFMAN, P. J. Superficial necrolytic dermatitis in 11 dogs with a history of phenobarbital administration (1995-2002). Journal of Veterinary Internal Medicine, United Kingdom, v. 18, n. 1, p. 65-74, 2004. Disponível em: <http://www.ncbi.nlm.nih.gov/pubmed/14765734>. Acesso em: 06 fev. 2012.

MÜLLER, P. B.; TABOADA, J.; HOSGOOD, G.; PARTINGTON, B. P.; VANSTEENHOUSE, J. L.; TAYLOR, H. W.; WOLFSHEIMER, K. J. Effects of long-term phenobarbital treatment on the liver in dogs. Journal of Veterinary Internal Medicine, United Kingdom, v. 14, n. 2, p. 165-171, 2000. Disponível em: $<$ http://www.ncbi.nlm.nih.gov/pubmed/10772488>. Acesso em: 06 fev. 2012.
POLDERVAART, J. H.; FAVIER, R. P.; PENNING, L. C.; VAN DEN INGH, T. S. G. A. M.; ROTHUIZEN, J. Primary hepatitis in dogs: a retrospective review (20022006). Journal of Veterinary Internal Medicine, United Kingdom, v. 23, n. 1, p. 72-80, 2009. Disponível em: $<$ http://www.ncbi.nlm.nih.gov/pubmed/19175724>. Acesso em: 06 fev. 2012.

PROPHET, E. B.; MILLS, B.; ARRINGTON, J. B.; SOBIN, L. H. AFIP Laboratory methods in histotechnology. American registry of Pathology. Washington: American Forces Institute of Pathology, 1996. $274 \mathrm{p}$.

SILVA, M. C.; FIGHERA, R. A.; BRUM, J. S.; GRAÇA, D. L.; KOMMERS, G. D.; IRIGOYEN, L. F.; BARROS, C. S. L. Cirrose hepática em cães: 80 casos (1965-2003). Pesquisa Veterinária Brasileira, Seropédica, v. 27, n. 11, p. 471-480, 2007.

STALKER, M. J.; HAYES, M. A. Liver and biliary system. In: MAXIE, M. G. (Ed.). Jubb, Kennedy and Palmer's. 5. ed. Philadelphia: Saunders Elsevier, 2007. v. 2, cap. 2, p. 297-388.

TAYLOR, S. M. Convulsões. In: NELSON, R. W.; COUTO, C. G. (Ed.). Medicina interna de pequenos animais. 4. ed. Rio de Janeiro: Elsevier, 2010. cap. 67, p. 1038-1048.

WOUTERS, A. T. B.; CASAGRANDE, R. A.; WOUTERS, F.; WATANABE, T. T. N.; BOABAID, F. M.; CRUZ, C. E. F.; DRIEMEIER, D. An outbreak of aflatoxin poisoning in dogs associated with aflatoxin B1-contaminated maize products. Journal of Veterinary Diagnostic Investigation, Athens, v. 25, p. 282-287, 2013. 